\title{
Arginine vasopressin facilitates reversal and impairs retention of a brightness discrimination in albino rats
}

\author{
BILL E. BECKWITH, TIMOTHY P. TINIUS, and DONALD H. MILLER \\ University of North Dakota, Grand Forks, North Dakota
}

\begin{abstract}
One microgram of arginine vasopressin (AVP) or a placebo was administered to male albino rats. Performance on the original learning, reversal learning, and retention of a black-white visual discrimination was assessed by means of both trials and errors to criterion. In Experiment 1, the rats were given AVP after original learning trials and prior to retention trials. In Experiment 2, the rats were given AVP prior to reversal trials and before retention trials. In Experiment 3, they were given AVP after original learning and then given a series of extinction trials. The results indicated that treatment with AVP after original learning trials facilitated performance during reversal learning but did not affect the rate of extinction. Treatment with AVP prior to reversal learning had no influence on performance during reversal learning. Finally, treatment with AVP impaired the 10-day retention of the discrimination learned during reversal training, that is, the black (+) discrimination. These findings are discussed in the context of their implications for the actions of vasopressin on selective attention and the possible independence of its actions on measures of attention and memory.
\end{abstract}

Current views suggest that in addition to its endocrine action on the kidneys, vasopressin may modulate learning and memory in both nonhuman subjects (De Wied \& Versteeg, 1979; van Wimersma Greidanus, van Ree, \& DeWied, 1983) and human subjects (Beckwith et al., 1982; Beckwith, Till, \& Schneider, 1984; Till \& Beckwith, 1985; Weingartner et al., 1981). The earliest evidence for a behavioral action of arginine vasopressin was provided by studies demonstrating that treatment with vasopressin reversed learning deficits induced by neurohypophysectomy (De Wied, 1969). Additional studies demonstrated that treatment with vasopressin produced resistance to extinction of active and passive avoidance behaviors (De Wied, 1969, 1971; De Wied \& Versteeg, 1979) and of appetitively motivated behavior (Ettenberg, Le Moal, Koob, \& Bloom, 1983) in intact animals. These findings led De Wied to suggest that vasopressin enhanced memory (De Wied \& Versteeg, 1979; van Wimersma Greidanus et al., 1983). This interpretation was further strengthened by the demonstration that treatment with vasopressin reversed amnesia induced by $\mathrm{CO}_{2}$ (Rigter, van Reizen, \& De Wied, 1974), puromycin (Walter, Hoffman, Flexner, \& Flexner, 1975), and hypothermia (Tinius, Beckwith, Wagner, Tinius, \& Traynor, 1986).

The memory hypothesis for vasopressin's actions on behavior has also received support from work with human subjects. Although the results of clinical trials have

\footnotetext{
We gratefully acknowledge the comments provided by Edward $S$. Halas and Thomas V. Petros on an earlier draft of this manuscript. Requests for reprints should be sent to Bill E. Beckwith, Psychology Department, University of North Dakota, Box 7187 University Station, Grand Forks, ND 58202.
}

been mixed (Tinklenberg \& Thornton, 1983), several studies have shown that treatment with vasopressin analogs enhanced memory performance both in healthy volunteer adult subjects (Beckwith et al., 1984; Till \& Beckwith, 1985; Weingartner et al., 1981) and in patients suffering from diabetes insipidus (Burish, Slonim, Davidson, More, \& Tower, 1982). A careful review of the literature indicated that part of the controversy over the actions of vasopressin on human memory may have stemmed from the use of insensitive tests of memory, failure to specify the degree of memory deficit, and/or the use of memory tasks not based on a specific theory of memory performance.

An alternative explanation is that memory enhancement, when it occurs, may be the result of vasopressin's actions on related processes, such as attention. Legros et al. (1978) were the first to demonstrate that vasopressin influenced attentional processes in human subjects. This action may, of course, contribute to enhancement of memory performance, but it may be separate from effects on processes such as consolidation and retrieval. Additional support for this explanation comes from a series of recent studies. Beckwith et al. (1982) demonstrated that a single intranasal administration of 1-desamino-D-arginine vasopressin (DDAVP) given to healthy young adult males enhanced the learning of a series of color-form discrimination shifts. Beckwith, Couk, and Till (1983) showed that treatment with DDAVP also enhanced attention during memory scanning to recall simple digits. Finally, Beckwith, Petros, Bergloff, and Staebler (1987) suggested that treatment with DDAVP facilitated the divided attentional processes necessary to integrate text in working memory by increasing attention to relevant as opposed 
to irrelevant details within narrative prose. In short, memory enhancement due to treatment with vasopressin may be a result of enhanced attention.

In a study aimed at assessing attention by using nonhuman subjects, Beckwith and Tinius (1985) administered arginine vasopressin to rats before both acquisition and reversal of a brightness discrimination in a modified $\mathrm{T}$ maze. In the initial stage of learning, the animals were required to learn a two-choice, simultaneous brightness discrimination with black and white doors as discriminative stimuli. The animals were first taught to approach and dislodge the white door to escape a mild footshock. Then, when they were able to enter the white door on 9 out of 10 consecutive trials, the task was altered so that entry through the black door allowed avoidance/escape. In other words, the animals were required to reverse their initial responses. Beckwith and Tinius reasoned that if vasopressin enhanced memory, as was suggested by its ability to retard extinction of learned behaviors, then treatment with vasopressin should retard the reversal of this brightness discrimination by making the response to the white stimulus door more difficult to relinquish. Although treatment with vasopressin did not influence performance during acquisition of the initial discrimination (when the white door was the $\mathrm{CS}+$ ), it did facilitate learning of the reversal discrimination (when the black door was the $\mathrm{CS}+$ ). They argued that treatment with vasopressin had enhanced attention.

The attentional analysis of these results follows the model presented by Sutherland and Mackintosh (1971). Accordingly, there are two behavioral requirements for mastery of a discrimination. Subjects must initially learn to attend to the relevant stimulus dimension that allows discrimination. (In the present study, subjects were expected to learn to suppress very strong position habits and, instead, attend to the brightness of the door-or, in terms of the model, to select and tune the appropriate sensory analyzer.) Thereafter, they can learn appropriate response attachments based on traditional reinforcement contingencies. Sutherland and Mackintosh postulated that these two processes develop somewhat independently, with analyzer strength accumulating at a different rate than response strength. This allows certain treatments (e.g., overtraining, treatment with vasopressin) to increase the strength of the attentional response to a greater degree than the choice response. Subsequently, animals that have received attention-enhancing treatments must extinguish only choice responses in order to relearn the correlation between the relevant cues and reinforcement, whereas nontreated animals must extinguish both attentional responses and choice responses in order to relearn the correlation between relevant cues and reinforcement. In short, treatment with vasopressin appears to strengthen selective attention to the relevant sensory analyzer and, thereby, enhance learning of the reversal shift.

The present study was done to replicate Beckwith and Tinius (1985) and to determine where in the training procedure (acquisition or reversal) vasopressin had its effects. According to the animal discrimination literature on reversal shifts, treatment (i.e., overtraining) is typically given after the animal has learned the original problem (Sutherland \& Mackintosh, 1971). Hence, the attentional response is strengthened by treatment presented after stimulus control is achieved. Furthermore, in reversal learning, the treatments are presented before training is given. It occurred to us that to approximate the methods used in the animal learning experiments, vasopressin could be administered after learning trials each day during original learning and before trials during reversal learning, which should allow more precise delineation of the timing of the effects and more closely isolate treatment to be "between" original learning and reversal. Daily treatments were given in a single dose $(1 \mu \mathrm{g})$ to more closely approximate the conditions used by Beckwith and Tinius (1985) and to avoid sedation or activation (Beckwith \& Tinius, 1984). The expectation was that posttrial administration of vasopressin during original learning would enhance reversal learning, an effect consistent with an attentional interpretation, whereas no prior prediction was made concerning the pretrial administration during reversal learning. Impaired performance during learning of the reversal problem, in either case, was expected to be consistent with a memory interpretation. Furthermore, a 10day retention trial was included to explore time-dependent alterations in memory after successful reversal.

\section{EXPERIMENT 1}

It is not clear from earlier work where vasopressin exerts its effect during the learning of a brightness discrimination and its reversal. Sara, Barnett, and Toussaint (1982) did all training in a single day, whereas Couk and Beckwith (1982) and Beckwith and Tinius (1985) extended training over several days. The present study was undertaken with the latter procedure so that results would more closely relate to the more traditional methods used in both animal discrimination learning (Sutherland \& Mackintosh, 1971) and other peptide studies that used the reversal learning paradigm (Beckwith \& Sandman, 1982; Beckwith \& Kastin, in press). Neither Couk and Beckwith (1982) nor Beckwith and Tinius (1985) attempted to determine where in the process of acquisition and reversal of the brightness discrimination vasopressin had its influence upon learning-treatment during original learning or treatment during reversal. The present experiment was done to determine if treatment with arginine vasopressin (AVP) given each day after original learning would influence performance during learning of the reversal problem. Furthermore, in an attempt to separate attentional from memoric processes, a 10-day delay was introduced (after successful completion of the reversal problem), after which animals were again treated with the peptide and required to perform the black discrimination to a $90 \%$ criterion. 


\section{Method}

\section{Subjects}

Twenty-one 70-day-old male albino rats (Holtzman Company, Madison, WI) used for this study were housed individually under controlled light-dark conditions (indirect lights on at 9:00 a.m., off at 9:00 p.m.). The animals were trained between 1:00 p.m. and 6:00 p.m. They were transported to and from the experimental room in their home cages and had access to food and water ad lib. The animals were left undisturbed for 6 days subsequent to their arrival at the laboratory and then handled individually for $10 \mathrm{~min}$ per day for 5 additional days before being adapted to the experimental apparatus.

\section{Apparatus}

The test apparatus used for all studies reported in this paper was a Thompson-Bryant discrimination box (Thompson \& Bryant, 1955). It was constructed from black Plexiglas and consisted of a startbox, a choice chamber, and a goalbox. Two discrimination doors, $89 \mathrm{~mm}$ square, at the end of the choice chamber were the only means of entering the goalbox. The two discrimination doors were separated by a partition that extended $7.5 \mathrm{~mm}$ into the choice chamber. The choice chamber and the startbox were separated by a guillotine door. The entire apparatus was covered by a three-sectioned, clear Plexiglas lid that prevented the animals from escaping. The apparatus was illuminated by a $100-\mathrm{W}$ bulb positioned $2 \mathrm{~m}$ above the center of the choice chamber. The discriminative stimuli used were solid white and solid black doors. During the pretraining phase, black and white, and vertically and horizontally striped doors were used to shape goalbox entry.

\section{Procedure}

The animals were randomly assigned to one of two groups: a group treated with AVP $(n=10)$ and a group treated with the placebo $(n=11)$. In a blind procedure, the experimental animals received $1 \mu \mathrm{g}$ (in $0.1 \mathrm{ml}$ of solution) AVP intraperitoneally (i.p.) immediately after the last trial each day throughout original learning. The placebo group received $0.1 \mathrm{ml}$ of the vehicle solution (saline) immediately after the last trial each day throughout acquisition of the white $(+)$ discrimination (i.e., original learning).

Pretraining. Each animal was permitted to explore the apparatus with free access to all chambers for $15 \mathrm{~min}$ on the first day of the pretraining phase. On the second day of pretraining, the animals were shaped to enter the goalbox by dislodging the doors of the choice chamber. This was done through a successive approximation procedure, which required that each animal be trained to dislodge the door in order to enter the goalbox. The animal was allowed to remain in the goalbox for $15 \mathrm{sec}$ before being removed for the next trial. The pretraining continued to a criterion of five consecutive errorless entries into the goalbox. An error was scored and the animal was given a discrete footshock of 0.5 -sec duration for each hesitation of $5 \mathrm{sec}$ in the choice chamber and/or for each turn toward the startbox after it entered the choice chamber. Throughout pretraining, original learning, reversal learning, and recall, the intensity of the shock was $0.5 \mathrm{~mA}$ and the duration of the shock was $0.5 \mathrm{sec}$.

Original learning. The visual discrimination task during original learning required the animal to run to the solid white door (positive stimulus), dislodge it, and enter the goalbox. The animal was allowed to remain in the goalbox for $15 \mathrm{sec}$ before being removed for the next trial. The solid black door (negative stimulus) was locked during this phase. The door positions were alternated according to a series of semirandom numbers (Gellerman, 1933). An error was scored and the animal was shocked if it approached within $7.5 \mathrm{~cm}$ of the negative stimulus, failed to approach either stimulus within $5 \mathrm{sec}$ after entering the choice chamber, or turned toward the start- box after entering the choice chamber. On error trials, the animals remained in the choice chamber until they escaped through the correct door. The criterion for learning was 9 out of 10 correct responses. Each animal was given 10 successive trials on each day of training. Immediately after the last trial each day, the animals were injected with either AVP or placebo and placed back in their home cages.

Reversal learning. On the day after the animals reached criterion on the original learning task, the positive stimulus was reversed so that the black door became the positive stimulus. During this phase, the solid white door remained locked and the door positions were alternated according to the same semirandom series. The animals were not injected during this phase of the experiment. The criteria used for acquisition of the response and the scoring of errors were the same as those used during the original learning phase.

Retention. Ten days after reaching criterion for reversal learning, the animals were again trained to run to the black door. Sixty minutes prior to training, the animals were injected i.p. with either AVP $(1 \mu \mathrm{g})$ or placebo. Half of the animals that had received AVP and half of the animals that had received placebo during original learning were given AVP during this experimental condition. The remaining animals were given placebo. The criteria used for acquisition of the response and the scoring of errors were the same as those used during reversal learning.

\section{Results and Discussion}

Both the number of trials to criterion and number of errors to criterion during original learning and reversal learning were analyzed by means of a 2 (treatment) $\times$ 2 (problem) analysis of variance (ANOVA) with repeated measures on the last factor.

For trials to criterion, as indicated in Table 1, there was a significant main effect for treatment $[F(1,19)=11.81$, $p=.003]$ and problem $[F(1,19)=75.27, p<.001]$ and a significant treatment $\times$ problem interaction $[F(1,19)=$ $6.36, p=.02]$. Likewise, errors to criterion showed a significant main effect of treatment $[F(1,19)=10.91, p$ $=.004]$ and problem $[F(1,19)=98.95, p<.001]$ and a significant treatment $\times$ problem interaction $[F(1,19)=$ $8.70, p=.009]$. The interactions were assessed by means

Table 1

Mean Trials to Criterion and Mean Errors to Criterion During Original Learning, Reversal Learning, and Retention for Animals Treated with Arginine Vasopressin After Original Learning and Prior to Retention

\begin{tabular}{|c|c|c|c|c|c|}
\hline \multirow[b]{2}{*}{ Group } & \multirow[b]{2}{*}{$n$} & \multicolumn{2}{|c|}{ Number of Trials } & \multicolumn{2}{|c|}{ Number of Errors } \\
\hline & & Mean & $S D$ & Mean & $S D$ \\
\hline \multicolumn{6}{|c|}{ Original Learning } \\
\hline Arginine Vasopressin & 10 & 57.8 & 13.8 & 23.7 & 6.2 \\
\hline Placebo & 11 & 66.9 & 25.2 & 27.8 & 9.2 \\
\hline \multicolumn{6}{|c|}{ Reversal Learning } \\
\hline Arginine Vasopressin & 10 & 131.0 & 41.9 & 66.9 & 15.2 \\
\hline Placebo & 11 & 200.1 & $54.8 *$ & 107.5 & 36.7 \\
\hline \multicolumn{6}{|c|}{ Retention } \\
\hline Arginine Vasopressin & 9 & 25.3 & $11.7^{*}$ & 7.7 & 5.7 \\
\hline Placebo & 12 & 14.6 & 8.3 & 2.7 & 2.9 \\
\hline
\end{tabular}


of the Newman-Keuls procedure with $\alpha<.05$. There was no significant effect of treatment with AVP during acquisition of the white-black discrimination. However, animals treated with AVP during original learning required fewer trials and made fewer errors in achieving criterion performance during reversal learning.

Both trials to criterion and number of errors to criterion during the 10-day test of retention were analyzed by means of a 2 (treatment during original learning) $\times 2$ (treatment during retention) ANOVA. Although treatment during original learning did not influence either the number of trials $(F<1.00)$ or number of errors $(F<1.00)$ to criterion, treatment with AVP 1 h prior to testing impaired retention as measured by both trials $[F(1,17)=$ $5.55, p=.03]$ and errors $[F(1,17)=6.19, p=.02]$ to criterion, as shown in Table 1 . There was no interaction between factors for either trials $(F<1.00)$ or errors $(F<1.00)$ to criterion.

These results indicated that animals treated with AVP during original learning of the white discrimination required fewer trials and made fewer errors to master the reversal problem than did a control group that received treatment with a placebo. This pattern of results indicates that treatment under these circumstances enhanced attention, an effect similar to that found by Beckwith and Tinius (1985). Therefore, the attentional facilitating effects of AVP appear to be a consequence of the emergence of the action of the peptide during the original learning of the discrimination and its manifestation during the learning of the reversal discrimination. These actions are independent of any influence on the learning of the original discrimination, which was not affected by treatment in the present study. This pattern is consistent with that found for the overtraining reversal effect, which, by the nature of this manipulation, also has no effect on learning the original discrimination. Furthermore, animals treated with AVP during a 10-day retention trial demonstrated poorer performance than their placebo-treated controls, an effect that was independent of initial treatment. In short, attention was facilitated by posttrial administration of AVP during original learning, whereas memory, as measured by a 10 -day retention test, was impaired. There were no state-dependent effects of treatment on memory performance.

\section{EXPERIMENT 2}

Experiment 1 demonstrated that treatment given during original learning produced enhanced performance of reversal learning. Experiment 2 was undertaken to investigate the actions of AVP on the reversal problem when treatment was given prior to each day's testing on the reversal learning problem but not during original learning. Although Beckwith and Tinius (1985) showed that treatment with AVP enhanced reversal learning, they failed to differentiate where in the sequence of training this effect occurs because they treated animals prior to both original learning and reversal learning. The follow- ing experiment was intended to evaluate the actions of AVP on reversal learning when animals had not received prior treatment during original learning. This experiment allowed inferences to be drawn about the locus of the treatment-induced facilitation of attentional processes (i.e., during original learning, during reversal learning, or both). A 10-day retention test was again used to attempt to replicate the unexpected finding of memory impairment reported in Experiment 1.

\section{Method}

\section{Subjects}

Twenty-one 70-day-old male Holtzman rats served as subjects. The animals in this experiment received the same treatment as those in Experiment 1 prior to any experimental treatment.

\section{Apparatus}

The same apparatus used in Experiment 1 was employed in Experiment 2 .

\section{Procedure}

The animals were randomly assigned to one of two groups: a group treated with AVP $(n=10)$ and a group treated with the placebo $(n=11)$. In a double-blind procedure the experimental animals received $1 \mu \mathrm{g}$ (in $0.1 \mathrm{ml}$ of solution) AVP i.p. $1 \mathrm{~h}$ prior to reversal learning trials each day. The other group received $0.1 \mathrm{ml}$ of vehicle (saline solution) $1 \mathrm{~h}$ prior to reversal learning trials each day. The pretraining, original learning, reversal learning, and retention phases of Experiment 2 were identical to those employed in Experiment 1 except that animals were injected with AVP or placebo $1 \mathrm{~h}$ prior to training in reversal learning rather than after original learning.

\section{Results and Discussion}

The results for number of trials to criterion and number of errors to criterion during original learning and reversal learning were analyzed by means of a 2 (treatment) $\times 2$ (problem) ANOVA with repeated measures on the last factor. As shown in Table 2, for trials to criterion there was a significant main effect for problem $[F(1,19)=106.42, p<.001]$, but there was neither a significant main effect for treatment $[F(1,19)=2.58$,

Table 2

Mean Trials to Criterion and Mean Errors to Criterion During Original Learning, Reversal Learning, and Retention for Animals Treated with Arginine Vasopressin Prior to Reversal Learning and Prior to Retention

\begin{tabular}{|c|c|c|c|c|c|}
\hline \multirow[b]{2}{*}{ Group } & \multirow[b]{2}{*}{$n$} & \multicolumn{2}{|c|}{ Number of Trials } & \multicolumn{2}{|c|}{ Number of Errors } \\
\hline & & Mean & $S D$ & Mean & $S D$ \\
\hline \multicolumn{6}{|c|}{ Original Learning } \\
\hline Arginine Vasopressin & 10 & 54.1 & 21.7 & 22.6 & 10.2 \\
\hline Placebo & 11 & 71.3 & 19.0 & 29.6 & 10.2 \\
\hline \multicolumn{6}{|c|}{ Reversal Learning } \\
\hline Arginine Vasopressin & 10 & 163.6 & 55.2 & 85.2 & 24.4 \\
\hline Placebo & 11 & 189.5 & 49.8 & 97.0 & 30.3 \\
\hline \multicolumn{6}{|c|}{ Retention } \\
\hline Arginine Vasopressìn & 10 & 24.2 & $15.8^{*}$ & 6.8 & 5.9 \\
\hline Placebo & 10 & 11.4 & 2.2 & 1.5 & 0.9 \\
\hline
\end{tabular}

${ }^{*} p<.05$. 
$p=.12]$ nor a significant treatment $\times$ problem interaction $(F<1.00)$. Likewise, errors to criterion did reveal a significant main effect for problem $[F(1,19)=152.25$, $p<.001]$, but revealed neither a significant main effect for treatment $[F(1,19)=1.60, p=.22]$ nor a significant treatment $\times$ problem interaction $(F<1.00)$.

Both trials to criterion and number of errors to criterion during the 10-day test of retention were analyzed by means of a 2 (treatment during reversal learning) $\times 2$ (treatment during retention) ANOVA. Although treatment during reversal learning did not influence either the number of trials $(F<1.00)$ or the number of errors $(F<$ $1.00)$ to criterion, treatment with AVP $1 \mathrm{~h}$ prior to testing impaired retention, as measured by both trials $[F(1,16)$ $=5.81 . p=.03]$ and errors $[F(1,16)=6.94, p<.02]$ to criterion, as shown in Table 2 . There was no interaction between factors for either trials $(F<1.00)$ or errors $(F<1.00)$ to criterion.

The results of Experiment 2 indicated that treatment with AVP given only during reversal learning had no effect on the performance of the animals. Furthermore, treatment with AVP at the dose used here did not sedate or otherwise impair the animals' performance, as would be inferred from poorer reversal. Apparently the enhancement of reversal learning (Beckwith \& Tinius, 1985; Experiment 1) caused by AVP occurred during acquisition of the initial discrimination. Furthermore, treatment with AVP again impaired performance on the 10-day retention test; this action was again independent of earlier treatment with the peptide.

\section{EXPERIMENT 3}

This experiment was undertaken to determine if the enhancement of reversal demonstrated in Experiment 1 was due to AVP's demonstrated ability to enhance resistance to extinction (Couk \& Beckwith, 1982; De Wied, 1971). Although this effect has been interpreted as evidence for memory enhancement, this is not the only interpretation possible. This experiment was designed to investigate an alternative explanation, which is described below.

It has been consistently demonstrated that overtraining on the original discrimination, a behavioral treatment that also enhances reversal learning, results in a greater resistance to extinction of the original discrimination (Sutherland \& Mackintosh, 1971). Therefore, it follows that if treatment with AVP (at the dose used here) enhanced resistance to extinction of the original learning by a process similar to that of overtraining, then the facilitation of learning the reversal might be a result of enhanced selective attention caused by the same process used to explain the overtraining-reversal effect.

\section{Method}

\section{Subjects}

Nineteen 70-day-old Holtzman albino rats were used for this study. The animals used in this experiment received the same treatment as those in the previous two experiments.

\section{Apparatus}

The apparatus used in Experiment 3 was described in Experiment 1 .

\section{Procedure}

The animals were randomly assigned to one of two groups: a group treated with AVP $(n=9)$ and a group treated with the placebo $(n=10)$. In a double-blind procedure, the experimental animals received $1 \mu \mathrm{g}$ (in $0.1 \mathrm{ml}$ of solution) AVP i.p. immediately after the last trial each day throughout original learning.

Pretraining. The pretraining phase of Experiment 3 was the same as that employed in Experiment 1.

Original learning. The original learning phase of Experiment 3 was the same as that employed in Experiment 1. The animals were injected with either AVP $(1 \mu \mathrm{g})$ or placebo i.p. immediately after the last trial each day.

Extinction. The animals received neither shocks nor AVP treatment during this phase of the experiment. Performance was evaluated by assessing the percent of correct trials to the white door on each day of testing. The solid black door remained locked, and the door positions were alternated according to the semirandom series. An unsuccessful trial was scored if the animal did not run to the white door within $30 \mathrm{sec}$ after the start of the trial.

\section{Results and Discussion}

For extinction, percent of trials correct per day were calculated for each animal and analyzed by means of a 2 (treatment) $\times 5$ (day) ANOVA with repeated measures on the last factor. As shown in Table 3 , there was a significant main effect for day $[F(4,68)=8.71, p<.001]$, but there was neither a significant main effect for treatment $(F<1.00)$ nor a significant treatment $\times$ day interaction $(F<1.00)$.

The results of Experiment 3 indicated that the enhancement of reversal learning found in Experiment 1 was not a result of an increased resistance to extinction. Posttrial treatment with AVP during original learning had no measurable influence on extinction in this study. Therefore, it appears that treatment with AVP enhances selective attention by means of a process or mechanism that differs from that used to explain the overtraining-reversal effect.

\section{GENERAL DISCUSSION}

Taken together, the results of the three experiments reported here suggest that posttrial treatment with AVP during acquisition of a brightness discrimination enhanced the learning of the reversal of that discrimination. Pretrial

Table 3

Mean Percentage of Correct Trials for Each Day of Extinction After Posttrial Administration of Arginine Vasopressin $(n=10)$ and Placebo $(n=9)$ During Original Learning

\begin{tabular}{cccccc}
\hline & \multicolumn{2}{c}{ Arginine Vasopressin } & & \multicolumn{2}{c}{ Placebo } \\
\cline { 2 - 3 } \cline { 5 - 5 } Day & Mean & $S D$ & & Mean & $S D$ \\
\hline 1 & 69.0 & 28.5 & & 72.2 & 28.6 \\
2 & 43.0 & 45.7 & & 61.1 & 35.5 \\
3 & 36.0 & 45.3 & & 47.8 & 36.3 \\
4 & 31.0 & 41.5 & & 44.4 & 35.7 \\
5 & 22.0 & 37.4 & & 38.9 & 32.2 \\
Overall & 40.2 & 25.8 & 52.9 & 35.3 \\
\hline
\end{tabular}


treatment with AVP during reversal learning had no consistent effect on performance. Furthermore, pretrial treatment with AVP impaired the 10-day retention of the problem learned during the reversal discrimination. The latter effect does not appear to be state-dependent, in that treatment with AVP during either original learning or reversal learning had no interaction with treatment during the test of retention.

The first major finding of this study, the facilitation of reversal learning after treatment with AVP within an aversively motivated discrimination task, is consistent with the results reported by Beckwith and Tinius (1985). Furthermore, attention appears to have been enhanced by AVP treatment during original learning. Treatment given prior to reversal learning did not influence performance. According to the model developed by Sutherland and Mackintosh (1971), there are two processes that underlie animal discrimination learning: (1) The animal must learn to attend to the relevant cues in the environment and (2) the animal must form appropriate response attachments. The former must occur before the latter for learning to be effective. This theory was developed in response to several studies that had demonstrated that, under some circumstances, overtraining on a brightness discrimination would facilitate reversal learning (e.g., Reid, 1953). The explanation offered by the attentional model is that overtraining enhances attention to the relevant stimulus dimension during original learning (i.e., brightness) and thereby enhances performance during the reversal of the discrimination. A human analog of the present experiment also indicated that treatment with vasopressin enhanced attention. Beckwith et al. (1982) demonstrated that male college students showed enhanced performance on a colorform discrimination test that paralleled the results of the present study. These studies, along with those of Legros et al. (1978) and Beckwith et al. (1983, 1987), suggest that treatment with vasopressin enhances attention.

The current findings suggest that vasopressin may facilitate attention independently of any action it may have on memory. An interesting result from the animal literature on discrimination learning is that overtraining enhances attention by increasing resistance to extinction. Vasopressin also has been shown to produce increased resistance to extinction (De Wied \& Versteeg, 1979). Indeed, this is one of the main rationales for the belief that treatment with AVP enhances memory. However, Experiment 3 demonstrated that posttrial treatment with AVP, under the conditions of this study, had no effect on the rate of extinction of the brightness discrimination. Therefore, it appears that (1) the mechanism by which vasopressin enhances attention is different from that by which overtraining enhances attention, despite the fact that both effects are engaged during manipulation of the conditions under which original learning occurs, and (2) AVP's failure to increase resistance to extinction implies that the mechanism by which it affects memory differs from that by which it affects attention. If the mechanisms were the same, then resistance to extinction should have resulted from treatment in both cases.

The second major finding of this study was that treatment with AVP impaired memory, as was demonstrated in both Experiments 1 and 2. This result is contrary to the findings of other studies, which have demonstrated that AVP treatment either facilitates memory (De Wied \& Versteeg, 1979; van Wimersma Greidanus et al., 1983) or has no effect on memory (Hostetter, Jubb, \& Kolowski, 1980; Sahgal, Keith, Wright, \& Edwardson, 1982). Of course, the method used in the present study was very different from those used by others, who have most often used passive avoidance paradigms as a memory task. However, it is interesting to note that studies using a similar paradigm to test the actions of $\mathrm{d}$-amphetamine have also shown a dissociation between the direction of effects produced by pharmacological treatment on attention and memory (e.g., Beckwith, Sandman, Alexander, Gerald, \& Goldman, 1974). An explanation that may be consistent with vasopressin's reported enhancement of memory is that administration of vasopressin prior to retrieval reactivated memories for both original and reversal learning. The conflict created by the reactivation of both memories at the same time induced poorer performance in the animals treated with vasopressin. But if that were the case, treatment should also have impaired reversal in Experiment 2, which it did not do. Maybe there is an interaction between treatment with vasopressin and the age of the memory (Beckwith et al., 1983), although this is purely speculative at the present time.

In summary, treatment with AVP enhanced selective attention via its action during original learning. Treatment during reversal learning did not influence performance. Subsequent treatment, regardless of whether attention was enhanced or not, impaired 10-day retention. It appears that treatment with vasopressin may influence both attention and memory independently. However, the mechanisms by which vasopressin affects these processes and the potential interaction between them remains to be more fully explored. For example, there is currently an intense debate centering on whether the actions of vasopressin are centrally mediated (e.g., De Wied \& Versteeg, 1979; van Wimersma Greidanus et al., 1983) or peripherally mediated (Ettenberg et al., 1983) via pressor actions. This issue needs further investigation to advance our understanding of the effects of vasopressin from a more physiological point of view. In the meantime, it may also be beneficial to focus our attention on the specific behavioral processes influenced by various vasopressin analogs, which may in turn suggest more specific ways to focus on the physiological mechanisms of their workings on learning, memory, and attention. Another question of interest in understanding the physiological basis for the actions of vasopressin concerns the time course of the hormone and its analogs. We are not aware of any current data that develop a time course of action to explain how treatment with a hormone that has a relatively brief half- 
life can alter performance $1 \mathrm{~h}$ after administration. It seems fairly obvious that the active hormone is no longer present, but the nature of the processes mediating these actions, whether central or peripheral, has not yet been described.

\section{REFERENCES}

Beckwith, B. E., Couk, D. I., \& TILL, T. S. (1983). Vasopressin analog influences the performance of males in a reaction time task. Peptides, 4, 707-709.

BecKWтTH, B. E., \& Kastin, A. J. (in press). Central actions of melanocyte-stimulating hormone (MSH). In A. Negro-Vilar \& P. M. Conn (Eds.), Peptide hormones: Effects and mechanisms of action. Boca Raton: CRC Press.

Beckwtih, B. E., Petros, T. V., Bergloff, P. J., \& Staebler, R. J. (1987). Vasopressin analog (DDAVP) facilitates recall of narrative prose. Behavioral Neuroscience, 101, 429-432.

Beckwtrh, B. E., Petros, T. V., Kanaan-Beckwith, S., Couk, D. I., HAUG, R. J., \& RYAN, C. (1982). Vasopressin analog (DDAVP) facilitates concept learning in human males. Peptides, 3, 627-630.

Beckwith, B. E., Sandman, C. A. (1982). Central nervous system and peripheral effects of ACTH, MSH and related neuropeptides. Peptides, 3, 411-420.

Beckwthi, B. E., Sandman, C. A., Alexander, W. D., Gerald, M. C., \& GoldmaN, H. (1974). d-Amphetamine effects on attention and memory in the albino and hooded rat. Pharmacology, Biochemistry \& Behavior, 2, 557-561.

BeCKWITh, B. E., TILL, R. E., \& SCHNEIDER, V. (1984). Vasopressin analog (DDAVP) improves memory in human males. Peptides, 5 , 819-822.

Beckwith, B. E., \& Tinius, T. P. (1984). The effects of 1, 5, 10, and 20 micrograms of arginine vasopressin on reversal of a brightness discrimination. Unpublished manuscript.

BECKwITH, B. E., \& Tinius, T. P. (1985). Vasopressin and vasotocin facilitate reversal of a brightness discrimination. Peptides, 6, 383-386.

Burish, T. B., Slonim, A. E., Davidson, E. S., More, G., \& Tower, A. (1982). Improved cognitive functioning in central diabetes insipidus following therapy with a vasopressin analog. Clinical Neurophysiology, 3, 13-15.

Covk, D. I., \& BECKwITH, B. E. (1982). Effects of desmopressin acetate (DDAVP) on the learning of a brightness discrimination. Peptides, 3, 521-526.

DE WIED, D. (1969). Effects of peptide hormones on behavior. In W. F. Ganong \& L. Martini (Eds.), Frontiers in neuroendocrinology (pp. 97140). New York: Oxford University Press.

DE WIED, D. (1971). Long-term effect of vasopressin on the maintenance of a conditioned avoidance response in rats. Nature, 232, 58-60.

DE WIED, D., \& VERSTEEG, H. G. (1979). Neuro-hypophyseal principles and memory. Federation Proceedings, 38, 2348-2358.

EttenberG, A., Le Moal, M., Кoob, G. F., \& Bloom, F. E. (1983). Vasopressin potentiation in the performance of a learned appetitive task: Reversal by a pressor antagonist analog of vasopressin. Pharmacology, Biochemistry \& Behavior, 18, 645-647.

Gellerman, L. W. (1933). Chance orders of altering stimuli in visual discrimination learning experiments. Journal of General Psychology, 42, 207-208.

Hostetter, G., Jubb, S. L., \& Kolowski, G. P. (1980). An inability of subcutaneous vasopressin to affect passive avoidance behavior. $\mathrm{Neu}$ roendocrinology, 30, 174-177.

legros, J. J., Gilot, P., Seron, X., Claesseus, J., Adams, A., Moeglen, J. M., Audibert, A., \& Berchier, P. (1978). Influence of vasopressin on learning and memory. Lancet, 1, 41-42.

REID, L. S. (1953). The development of noncontinuity behavior through continuity learning. Journal of Experimental Psychology, 46, 107-112.

Rigter, H., van Reizen, H., \& De Wied, D. (1974). The effects of $\mathrm{ACTH}$ and vasopressin analogues on $\mathrm{CO}_{2}$ induced retrograde amnesia in rats. Physiology \& Behavior, 13, 381-388.

Sahgal, A., KeITH, A. B., Wright, C., \& EdWardson, J. A. (1982). Failure of vasopressin to enhance memory in a passive avoidance task in rats. Neuroscience Letters, 28, 87-92.

SARa, S. J., BarneTt, J., \& Toussaint, P. (1982). Vasopressin accelerates appetitive discrimination learning and impairs its reversal. Behavioural Processes, 7, 157-167.

Sutherland, N. S., \& MaCkintosh, N. J. (1971). Mechanisms of animal discrimination learning. New York: Academic Press.

Thompson, R., \& BRyant, J. H. (1955). Memory as affected by the relevant receptor. Psychological Reports, 1, 393-400.

TILL, R. E., \& BECKWTTH, B. E. (1985). Sentence memory affected by vasopressin analog (DDAVP) in cross-over experiment. Peptides, 6, 397-402.

TinkLenberG, J. R., \& Thornton, J. E. (1983). Neuropeptides in geriatric psychopharmacology. Psychopharmacology Bulletin, 19, 199-211.

Tinius, T. P., Beckwith, B. E., Wagner, N., Tinius, K. A., \& TRAYNER, M. M. (1986). Differential actions of arginine vasopressin and alpha-melanocyte-stimulating hormone on reactivation of memory after hypothermia-induced amnesia. Physiological Psychology, 14, 15-22.

van Wimersma Greidanus, T. B., van Ree, J. M., \& De Wied, D. (1983). Vasopressin and memory. Pharmacology \& Therapeutics, 20 , 437-458.

Walter, R., Hoffman, P. L., Flexner, J. B., \& Flexner, L. B. (1975). Neurohypophyseal hormones, analogs and fragments: Their effect on puromycin-induced amnesia. Proceedings of the National Academy of Sciences (USA), 72, 4180-4184.

Weingartner, H., Gold, P., Ballenger, J. C., Smallberg, S. A., Summers, R., Rubinov, D. R., Post, R. M., \& Goodwin, F. K. (1981). Effects of vasopressin on human memory functions. Science, 211, 601-603.

(Manuscript received June 19, 1987; revision accepted for publication August 25, 1987.) 